\title{
ANTIMICROBIAL SENSITIVITY OF GRAM-NEGATIVE BACTERIA WITH BETA LACTAM /BETA LACTAMASE INHIBITOR COMBINATION OF DRUGS IN COMPARISON TO OTHER ANTIBIOTICS IN TERTIARY CARE CENTER
}

\author{
N. Rammurugan ${ }^{1}$, R. Sasireha ${ }^{2}$
}

${ }^{1}$ Associate Professor, Department of Microbiology, Government Sivagangai Medical College, Sivagangai, Tamilnadu, India.

${ }^{2}$ Assistant Professor, Department of Microbiology, Government Sivagangai Medical College, Sivagangai, Tamilnadu, India.

ABSTRACT
BACKGROUND
Objectives of the study were- 1. isolation and speciation of Gram-negative bacteria from urine, pus sample, wound swab, blood,
tissue, BAL and other body fluids. 2. To identify bacteria most commonly isolated in ICU and Non-ICU Samples. 3. To compare
sensitivity and resistant patterns of beta lactam and beta lactamase inhibitor combination of drugs, carbapenem, and
aminoglycosides in various Gram-negative bacterial isolates.

Bacterial isolates are compared with MIC testing (E test) for Cefepime Tazobactam (CPT) and disc diffusion method.

\section{MATERIALS AND METHODS}

Gram negative bacterial pathogens from various clinical samples (urine, blood, broncho-alveolar lavage [BAL], other body fluids (bile, endometrial secretions), pus, wound swab, sputum and vaginal swab) were included in this study. The Isolates were identified with a battery of standard biochemical tests. Cefepime/tazobactam (CPT) $(30 / 10 \mu \mathrm{g}$ HiMedia, Mumbai) against various bacterial isolates and their susceptibility were compared with other $\beta$-lactam/ $\beta$-lactamase inhibitor combinations like piperacillin/ tazobactam (PTZ), cefoperazone/sulbactam (CFS), carbapenems [imipenem (IMP), meropenem (MRP) and Amikacin]. MIC determination paper strip which is coated with Cefepime/Tazobactam on a single paper strip in a concentration gradient capable of showing MICs in the range of $0.016 \mathrm{mcg} / \mathrm{ml}$ to $256 \mathrm{mcg} / \mathrm{ml}$, on testing against the test organism.

\section{RESULTS}

A total of 130 isolates from patients attending the tertiary care teaching hospital were included in the study. Most common age groups of isolation of Gram-negative bacilli was 40-60 yrs. and above 60 yrs. Out of 130 isolates isolated, 68 isolates were from male patients and 53 isolates were from female patients, 7 isolates were from male children and 2 isolates were from female children. Out of 130 isolates isolated, most common area of isolation of Gram-negative bacilli were from Inpatient (IP) from various wards, Out Patient (OP) and ICU. Out of 130 isolates isolated, most common samples were from urine, blood, pus and wound swab. Out of 130 samples isolated, 43\% were from Escherichia coli, 24\% were from Klebsiella pneumoniae, 18\% were from Pseudomonas aeruginosa, 5\% were from Enterobacter species and 3\% were from Proteus species. Among 43\% E. coli isolates, the sensitivity pattern towards, Cefepime Tazobactam was 90\%, Cefepime - 32\%, Piperacillin Tazobactam -75\%, Cefoperazone sulbactam -71\%, Carbapenem- 91\%, and Amikacin- 92\%. Klebsiella Pneumoniae (24\%) showed susceptibility of 42\% on Cefepime Tazobactam, Cefepime-16\%, Piperacillin Tazobactam -48\%, Cefoperazone sulbactam $-46 \%$, Carbapenem-55\%, and Amikacin 55\%. Pseudomonas aeruginosa (18\%) showed highest susceptibility of $87 \%$ Sensitivity to Cefepime Tazobactam, Cefepime, Carbapenem and Amikacin followed by 70\% Sensitivity to Piperacillin Tazobactam and Cefoperazone sulbactam. Proteus spp. showed $100 \%$ susceptibility to Cefepime Tazobactam, Cefoperazone sulbactam, Carbapenem and Amikacin followed by $80 \%$ sensitivity to Cefepime, Piperacillin Tazobactam. Citrobacter spp. showed highest percentage of $100 \%$ susceptibility to Cefepime Tazobactam, Cefepime followed by $75 \%$ sensitivity to CFS, PIT, Carbapenem and Amikacin. Acinetobacter spp. Showed 50\% sensitivity to Carbapenem and Amikacin respectively. E. Coli showed 68\% resistant to Cefepime. Klebsiella showed resistant to 58\%-Cefepime Tazobactam, 84\%-, Cefepime, 52\%-Piperacillin Tazobactam and 54\% Cefoperazone sulbactam. Acinetobacter showed 100\% resistant to Cefepime Tazobactam, Cefepime, Piperacillin Tazobactam and Cefoperazone sulbactam. Out of 130 Samples, Cefepime Tazobactam showed highest sensitivity to E. Coli, Enterobacter, Pseudomonas, Proteus, Serratia and Citrobacter. It showed resistant to Klebsiella and Acinetobacter.

\section{CONCLUSION}

This study showed a high level of antibiotic resistance among Gram-negative bacilli, particularly E. coli, K. pneumoniae and Acinetobacter spp. to the third generation cephalosporins. Cefepime Tazobactam is highly sensitive than Cefoperazone Sulbactam, Ceftazidime Sulbactam and Piperacillin Tazobactam. Cefepime, Tazobactam can be used to limit the Carbapenem usage in hospitals. E test and Antibiotic disc diffusion test showed equally sensitivity and resistant patterns.

\section{KEY WORDS}

Cefepime Tazobactam, Gram Negative Bacteria, E Test.

HOW TO CITE THIS ARTICLE: Rammurugan N, Sasireha R. Antimicrobial sensitivity of gram-negative bacteria with beta lactam /beta lactamase inhibitor combination of drugs in comparison to other antibiotics in tertiary care center. J. Evolution Med. Dent. Sci. 2019;8(07):416-420, DOI: 10.14260/jemds/2019/92 
'Financial or Other Competing Interest': None.

Submission 17-01-2019, Peer Review 07-02-2019,

Acceptance 09-02-2019, Published 18-02-2019.

Corresponding Author:

Dr. R. Sasireha,

Assistant Professor,

Department of Microbiology,

Government Sivagangai Medical College,

Sivagangai, Tamilnadu, India.

E-mail: rammddo@yahoo.co.in

DOI: $10.14260 /$ jemds $/ 2019 / 92$

\section{(c) (i) $\odot$}

\section{BACKGROUND}

Gram negative bacilli (GNB) continue to be an important cause of health care associated infections. Antimicrobial resistance among these bacilli is increasing on a worldwide basis, especially resistance against $\beta$ lactam antibiotics due to the development of $\beta$ lactamase enzymes. As a result, it creates therapeutic failure or increases the morbidity among the patients. ${ }^{1}$ Cefepime is a fourth-generation cephalosporin antibiotic. Cefepime has an extended spectrum of activity against Gram-positive and Gram-negative bacteria, with greater activity against both types of organism than thirdgeneration agents. It is stable against AmpC \& OXA. Cefepime has good activity against important pathogens including Pseudomonas aeruginosa, Staphylococcus aureus, and multiple drug-resistant Streptococcus pneumoniae. Cefepime is usually reserved to treat moderate to severe nosocomial pneumonia, infections caused by multiple drug-resistant microorganisms. ${ }^{2}$ Tazobactam inhibits the action of bacterial $\beta$-lactamases, especially those belonging to the SHV-1 and TEM groups. It can be added to certain antibiotics to make them less vulnerable to bacteria's antimicrobial resistance. Tazobactam is combined with the fourth-generation cephalosporin Cefepime. ${ }^{3}$ Cefepime and Tazobactam are prescribed for the treatment of uncomplicated skin and skin structure infections, uncomplicated and complicated urinary tract infections (UTI) and complicated intra-abdominal infections in adults and children, and it is also approved for use for empirical therapy for febrile neutropenic patients. ${ }^{4}$ Cefepime and Tazobactam are indicated as a parenteral therapy for the treatment of various moderate to severe forms of infections due to susceptible beta-lactamase producing microbial organisms.5,6 Cefepime and Tazobactam combination are particularly indicated if the Cefepime monotherapy is ineffective. Considering these therapeutic challenges, this study was aimed to compare the in-vitro antimicrobial effect of carbapenems, piperacillin/Tazobactam and cefoperazone/sulbactam with Cefepime/ tazobactam - a new $\beta$-lactam/ $\beta$-lactamase inhibitor combination. This study was done to evaluate the current status of the drugs which will help the clinicians to prescribe the appropriate drugs to the patients to reduce their hospital stay and cost of treatment.

\section{MATERIALS AND METHODS}

\section{Type of Study}

Descriptive study.

\section{Settings}

The study was conducted in a Department of Microbiology, Government Sivagangai Medical College, Sivagangai.

\section{Sample Size}

130 isolates received from various Patients Samples with different Clinical condition.

\section{Study Period}

From July to December 2018.

\section{Inclusion Criteria}

Newly admitted patients without any antibiotic treatment.

\section{Exclusion Criteria}

Patients already with antibiotic treatments. Newborn child. Repeated isolates from the same patients.

\section{Methodology}

A total of 130 non-repetitive, consecutive aerobic Gramnegative bacterial pathogens from various clinical samples (Urine, Blood, broncho-alveolar lavage [BAL], other body fluids (Bile, endometrial secretions), Pus, Wound Swab, Sputum and Vaginal Swab) were included in this study. The samples were transported to the lab and streaked on MacConkey Agar and Blood Agar medium. After the incubation period at $37^{\circ} \mathrm{C}$, the culture plates were examined, and bacterial isolates were observed by Grams Staining and motility test. Isolates were identified with a battery of standard biochemical tests ${ }^{7}$ Cefepime/tazobactam (CPT) $(30 / 10 \mu \mathrm{g}$ Hi Media, Mumbai) against various bacterial isolates and to compare its susceptibility with other $\beta$ lactam/ $\beta$-lactamase inhibitor combination like Piperacillin/ tazobactam (PTZ), Cefoperazone/sulbactam (CFS), Carbapenems [imipenem (IMP), meropenem (MRP) and Amikacin]. The antibiotic sensitivity was done with Mueller Hinton agar according to Kirby- Bauer technique. ${ }^{8}$ Cefepime/tazobactam interpretative criteria was not available, Cefoperazone and Cefepime zone size as per CLSI 2018 was used to interpret these two drug combinations (CLSI 2018). MIC determination paper strip which is coated with Cefepime/Tazobactam on a Single paper strip in a concentration gradient manner, capable of showing MICs in the range of $0.016 \mathrm{mcg} / \mathrm{ml}$ to $256 \mathrm{mcg} / \mathrm{ml}$, on testing against the test organism. Enterobacteriaceae group of organisms for which the Cefepime Tazobactam zone diameters below $2 \mathrm{~mm}$ are considered susceptible, 4-8 $\mathrm{mm}$ are Intermediate sensitivity, $>16 \mathrm{~mm}$ are Resistant. Non-fermenters group of organisms for which the Cefepime Tazobactam zone diameters below $8 \mathrm{~mm}$ are considered susceptible, $16 \mathrm{~mm}$ are Intermediate sensitivity, $>32 \mathrm{~mm}$ are Resistant. ${ }^{9}$ ATCC control strains, Escherichia coli ATCC 25922 and Pseudomonas aeruginosa ATCC 27853 were used as controls.

The study was approved by the Institutional ethical committee.

\section{RESULTS}

\begin{tabular}{|c|c|}
\hline Age in Years & Numbers $(n=130)$ \\
\hline $1-12$ & 9 \\
\hline $13-40$ & 23 \\
\hline $40-60$ & 42 \\
\hline 61 and Above & 56 \\
\hline Total & 130 \\
\hline \multicolumn{2}{|c|}{ Table 1. Age Wise Distribution of Samples } \\
\hline
\end{tabular}

A total of 130 isolates from patients attending the tertiary care teaching hospital. Most common age group of isolation of Gram-negative bacilli were from 40-60 yrs. and above 60 yrs. [Table. 1] 


\begin{tabular}{|c|c|}
\hline Sex & Numbers (n=130) \\
\hline Male & 68 \\
\hline Female & 53 \\
\hline Male Child & 7 \\
\hline Female Child & 2 \\
\hline \multicolumn{2}{|c|}{ Total } \\
\hline \multicolumn{2}{|c|}{ Table 2. Sex Wise Distribution of Samples } \\
\hline
\end{tabular}

Out of 130 isolates isolated, 68 isolates were from male patients and 53 isolates were from female patients. 7 isolates were from male children and 2 isolates were from female children. [Table. 2]

\begin{tabular}{|c|c|}
\hline Places & Numbers(n=130) \\
\hline $\mathrm{OP}$ & 43 \\
\hline IP & 69 \\
\hline ICU & 16 \\
\hline PICU & 1 \\
\hline CTICU & 1 \\
\hline Total & 130 \\
\hline
\end{tabular}

Out of 130 isolates isolated, most common area of isolation of Gram-Negative Bacilli were from Inpatient (IP) from various wards, Out Patient (OP) and ICU. [Table. 3]

\begin{tabular}{|c|c|}
\hline Samples & Numbers $(n=130)$ \\
\hline Urine & $39(30 \%)$ \\
\hline Blood & $16(12 \%)$ \\
\hline PUS & $31(24 \%)$ \\
\hline Sputum & 3 \\
\hline BAL & 6 \\
\hline Other Body fluids & 2 \\
\hline Tissue & 5 \\
\hline Vaginal Swab & 2 \\
\hline Wound Swab & $26(20 \%)$ \\
\hline Total & 130 \\
\hline \multicolumn{2}{|c|}{ Table 4. Samples Isolation } \\
\hline
\end{tabular}

Out of 130 isolates isolated, most common Samples were from Urine, Blood, Pus and Wound Swab. [Table. 4]

\begin{tabular}{|c|c|}
\hline $\begin{array}{c}\text { Organism in } \\
\text { Percentage }\end{array}$ & Numbers $(n=130)$ \\
\hline E. Coli $(43 \%)$ & $\begin{array}{c}\text { 56: - Urine-30, Blood-6, Wound } \\
\text { swab-7, Pus-9, BAL-1, Tissue-1, } \\
\text { Other fluids-1, Vaginal Swab-1 } \\
\end{array}$ \\
\hline $\begin{array}{l}\text { Klebsiella pneumonia } \\
\qquad(24 \%)\end{array}$ & $\begin{array}{c}\text { 31: - Urine-5, Blood-3, Bal-4, Pus-7, } \\
\text { Wound Swab-6, Sputum-1, Tissue-3, } \\
\text { Other Fluids-1, Vaginal Swab-1 }\end{array}$ \\
\hline $\begin{array}{l}\text { Enterobacter } \\
\text { Species }(5 \%)\end{array}$ & $\begin{array}{l}\text { 7: - Urine-1, Blood-2, Wound Swab-1, } \\
\text { Pus-3 }\end{array}$ \\
\hline Proteus species (3\%) & $\begin{array}{l}\text { 5: - Pus-2, Blood-1, Tissue-1, Wound } \\
\text { Swab-1 }\end{array}$ \\
\hline $\begin{array}{c}\text { Pseudomonas } \\
\text { aeruginosa }(18 \%)\end{array}$ & $\begin{array}{l}\text { 23: - Urine-2, Wound Swab-8, Pus-8, } \\
\text { Sputum-2, Blood-2, Bal-1 }\end{array}$ \\
\hline Serratia marcescens & 2: -Blood-2 \\
\hline Acinetobacter & 2: - Wound Swab-2 \\
\hline Citrobacter koseri $(2 \%)$ & 4: - Pus-2, Wound Swab-1, Urine-1 \\
\hline Total & 130 \\
\hline \multicolumn{2}{|c|}{$\begin{array}{l}\text { Table 5. Gram Negative Bacteria's Isolated from Various } \\
\text { Samples }\end{array}$} \\
\hline
\end{tabular}

Table 5, Out of 130 samples isolated, $43 \%$ were from Escherichia Coli, 24\% were from Klebsiella Pneumoniae, 18\% were from Pseudomonas aeruginosa, 5\% were from Enterobacter Species and 3\% were from Proteus Species.

\begin{tabular}{|c|c|c|c|c|c|c|}
\hline 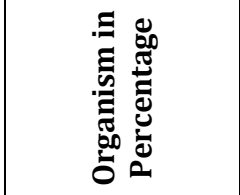 & 导 & 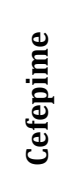 & 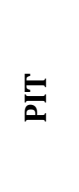 & 岳 & 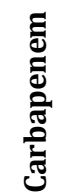 & 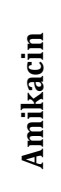 \\
\hline & $\begin{array}{c}\text { Sen } \\
\%\end{array}$ & $\begin{array}{c}\text { Sen } \\
\%\end{array}$ & $\begin{array}{c}\text { Sen } \\
\%\end{array}$ & $\begin{array}{c}\text { Sen } \\
\%\end{array}$ & $\begin{array}{c}\text { Sen } \\
\%\end{array}$ & $\begin{array}{c}\text { Sen } \\
\%\end{array}$ \\
\hline E. Coli (43) & 90 & 32 & 75 & 71 & 91 & 92 \\
\hline \begin{tabular}{|c|} 
Klebsiella \\
Pneumoniae (24)
\end{tabular} & 42 & 16 & 48 & 46 & 55 & 55 \\
\hline $\begin{array}{c}\text { Pseudomonas } \\
\text { aeruginosa (18) }\end{array}$ & 87 & 86 & 70 & 70 & 87 & 87 \\
\hline $\begin{array}{c}\text { Enterobacter } \\
\text { Species (5) }\end{array}$ & 100 & 100 & 100 & 100 & 100 & 100 \\
\hline $\begin{array}{c}\text { Proteus Species } \\
\text { (3) }\end{array}$ & 100 & 80 & 80 & 100 & 100 & 100 \\
\hline $\begin{array}{l}\text { Citrobacter } \\
\text { Species (2) }\end{array}$ & 100 & 100 & 75 & 75 & 75 & 75 \\
\hline Serratia & 100 & 100 & 100 & 100 & 100 & 100 \\
\hline $\begin{array}{l}\text { Acinetobacter } \\
\text { Species }\end{array}$ & 0 & 0 & 0 & 0 & 50 & 50 \\
\hline & & & & & & \\
\hline
\end{tabular}

Table 6, among 43\% E. coli isolates, the sensitivity pattern towards, Cefepime Tazobactam was $90 \%$, Cefepime - 32\%, Piperacillin Tazobactam $-75 \%$, Cefoperazone sulbactam 71\%, Carbapenem- 91\%, and Amikacin- 92\%. Klebsiella Pneumoniae (24\%) showed susceptibility of $42 \%$ on Cefepime Tazobactam, Cefepime-16\%, Piperacillin Tazobactam $-48 \%$, Cefoperazone sulbactam $-46 \%$, Carbapenem-55\%, and Amikacin 55\%. Pseudomonas aeruginosa (18\%) showed highest susceptibility to $87 \%$ Sensitivity to Cefepime Tazobactam, Cefepime, Carbapenem and Amikacin followed by $70 \%$ Sensitivity to Piperacillin Tazobactam and Cefoperazone sulbactam. Proteus spp. showed $100 \%$ susceptibility to Cefepime Tazobactam, Cefoperazone sulbactam, Carbapenem and Amikacin followed by $80 \%$ sensitivity to Cefepime, Piperacillin Tazobactam. Citrobacter spp. showed highest percentage of $100 \%$ susceptibility to Cefepime Tazobactam, Cefepime followed by $75 \%$ sensitivity to CFS, PIT, Carbapenem and Amikacin. Acinetobacter spp. Showed 50\% sensitivity to Carbapenem and Amikacin respectively.

\begin{tabular}{|c|c|c|c|c|c|c|}
\hline 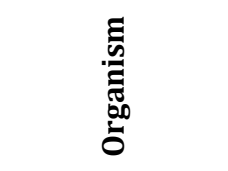 & 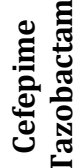 & 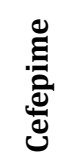 & 5 & 点 & 茪 & 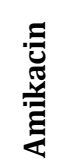 \\
\hline & $\begin{array}{c}\text { Res } \\
\%\end{array}$ & $\begin{array}{c}\text { Res } \\
\%\end{array}$ & $\begin{array}{c}\text { Res } \\
\%\end{array}$ & $\begin{array}{c}\text { Res } \\
\%\end{array}$ & $\begin{array}{c}\text { Res } \\
\%\end{array}$ & $\begin{array}{c}\text { Res } \\
\%\end{array}$ \\
\hline E. coli & 10 & 68 & 25 & 29 & 9 & 8 \\
\hline $\begin{array}{c}\text { Klebsiella } \\
\text { pneumoniae }\end{array}$ & 58 & 84 & 52 & 54 & 45 & 45 \\
\hline $\begin{array}{c}\text { Pseudomonas } \\
\text { aeruginosa }\end{array}$ & 13 & 14 & 30 & 30 & 13 & 13 \\
\hline Proteus Species & 0 & 20 & 20 & 0 & 0 & 0 \\
\hline $\begin{array}{c}\text { Citrobacter } \\
\text { Species }\end{array}$ & 0 & 0 & 25 & 25 & 25 & 25 \\
\hline $\begin{array}{l}\text { Acinetobacter } \\
\text { Species }\end{array}$ & 100 & 100 & 100 & 100 & 50 & 50 \\
\hline
\end{tabular}


E. Coli showed $68 \%$ resistant to Cefepime. Klebsiella showed resistant to 58\%-Cefepime Tazobactam, 84\%-, Cefepime, 52\%-Piperacillin Tazobactam and 54\% Cefoperazone sulbactam. Acinetobacter showed 100\% resistant to Cefepime Tazobactam, Cefepime, Piperacillin Tazobactam and Cefoperazone sulbactam. (Table. 7).

\begin{tabular}{|c|c|c|c|c|}
\hline $\begin{array}{c}\text { Cefepime } \\
\text { Interpretation }\end{array}$ & S & SDD & R & ATCC - QC \\
\hline Enterobacteriaceae & $<2$ & $4 \_8$ & $>=16$ & Escherichia coli \\
\hline
\end{tabular}

Table 8. MIC of Cefepime Tazobactam with E Strips Method (HiMedia) - CLSI 2018.

\begin{tabular}{|c|c|c|}
\hline Organism & Cefepime Tazobactam MIC (E Test \%) \\
\hline & Sen \% & Res \% \\
\hline E. Coli & 90 & 10 \\
\hline Klebsiella & 45 & 55 \\
\hline Pseudomonas & 87 & 13 \\
\hline Enterobacter & 100 & 0 \\
\hline Proteus Spp. & 100 & 0 \\
\hline Citrobacter & 100 & 0 \\
\hline Acinetobacter & 0 & 100 \\
\hline Serratia & 100 & 0 \\
\hline \multicolumn{3}{|c|}{ Table 9 } \\
\hline
\end{tabular}

Out of 130 Samples, Cefepime Tazobactam showed highest sensitivity to E. Coli, Enterobacter, Pseudomonas, Proteus, Serratia and Citrobacter. It showed resistant to Klebsiella and Acinetobacter. (Table 8).

\section{DISCUSSION}

In Our Study, Infections due to Gram negative organisms are commonly isolated in the clinical Sample. Similar observations have been made by others; Bhat et al and Shrestha et al.10,11

In this study, E. coli, Klebsiella spp, and P. aeruginosa are the most commonly isolated organism. The bacterial isolates were collected mainly from patients with urinary tract infections (30\%), Pus and wound swab (44\%) and bloodstream infections (12\%) correlates with Veeraraghavan B et al. ${ }^{12}$

In this study, among $43 \%$ E. coli isolates, the sensitivity pattern towards, Cefepime Tazobactam was $90 \%$, Cefepime $32 \%$, Piperacillin Tazobactam $-75 \%$, Cefoperazone sulbactam -71\%, Carbapenem- 91\%, and Amikacin- 92\%. Klebsiella Pneumoniae (24\%) showed susceptibility to $42 \%$ on Cefepime Tazobactam, Cefepime-16\%, Piperacillin Tazobactam -48\%, Cefoperazone sulbactam -46\%, Carbapenem-55\%, and Amikacin 55\%. Pseudomonas aeruginosa (18\%) showed highest susceptibility of $87 \%$ Sensitivity to Cefepime Tazobactam, Cefepime, Carbapenem and Amikacin followed by $70 \%$ Sensitivity to Piperacillin Tazobactam and Cefoperazone sulbactam. Proteus spp. showed $100 \%$ susceptibility to Cefepime Tazobactam, Cefoperazone sulbactam, Carbapenem and Amikacin followed by $80 \%$ sensitivity to Cefepime, Piperacillin Tazobactam. Citrobacter spp. showed highest percentage of $100 \%$ susceptibility towards Cefepime Tazobactam, Cefepime followed by $75 \%$ sensitivity to CFS, PIT, Carbapenem and Amikacin. Acinetobacter spp. Showed $50 \%$ sensitivity to Carbapenem and Amikacin respectively.

Addition of tazobactam increased the susceptibility of from $32 \%$ to $90 \%$ in E. coli, from 16 to $41.0 \%$ in Klebsiella, from 86.0 to $87 \%$ in Pseudomonas and Proteus from $80 \%$ to $100 \%$. This study highly correlates with Abdul Kafur et al. ${ }^{13}$

Cefepime/tazobactam having better coverage than Cefepime, Cefoperazone-sulbactam, Piperacillin Tazobactam and Ceftazidime Tazobactam correlates with my previous sudy. 14

Cefepime tazobactam $(30 / 10 \mu \mathrm{g})$ combination was found to be very effective against many ESBL producing Gram negative organism. As susceptibility rates to Cefepime are severely decreasing, Cephalosporins should not be a useful for treatment.

In this study, E. Coli showed $68 \%$ resistant to Cefepime. Klebsiella showed resistant to 58\%-Cefepime Tazobactam, 84\%-, Cefepime, 52\%-Piperacillin Tazobactam and 54\% Cefoperazone sulbactam. Acinetobacter showed 100\% resistant to Cefepime Tazobactam, Cefepime, Piperacillin Tazobactam and Cefoperazone sulbactam correlates with Bhat $\mathrm{V}$ et al. ${ }^{15}$

Gram negative isolates were most frequently sensitive to Carbapenems and Cefepime Tazobactam followed by aminoglycosides in this study.

The high rate of Enterobacteriaceae resistance to Carbapenem is a serious concern. At the same time reducing Carbapenem resistance in Pseudomonas is encouraging correlates with Dalal p et al. 16

Cefepime Tazobactam E test (Mic) showed highest sensitivity to E. Coli, Enterobacter, Pseudomonas, Proteus, Serratia and Citrobacter. It showed resistant to Klebsiella and Acinetobacter. Same findings are confirmed with disc diffusion method also.

\section{CONCLUSION}

This study showed a high level of antibiotic resistance among Gram-negative bacilli, particularly E. coli, K. pneumoniae and Acinetobacter spp. to the third generation cephalosporins. Cefepime Tazobactam is more effective than Cefoperazone Sulbactam, Ceftazidime Sulbactam and Piperacillin Tazobactam. Cefepime Tazobactam can be used us to limit the Carbapenem usage in hospitals. E test and Antibiotic disc diffusion test showed equally sensitivity and resistant patterns.

\section{Limitations}

This is a single center study.

\section{ACKNOWLEDGEMENT}

We would like to thank the Dean, Government Sivagangai Medical College, Sivagangai for his help and guidance while conducting this Study. Authors acknowledge the help of technicians and co-staff who have helped to conduct this study successfully.

\section{REFERENCES}

[1] Sood S. Comparative evaluation of the in-vitro activity of six $\beta$-lactam/ $\beta$-lactamase inhibitor combinations against Gram Negative Bacilli. J Clin Diagn Res 2013;7(2):224-8.

[2] Chapman TM, Perry CM. Cefepime: a review of its use in the management of hospitalized patients with pneumonia. Am J Respir Med 2003;2(1):75-107. 
[3] Yang Y, Rasmussen BA, Shlaes DM. Class A betalactamases-enzyme-inhibitor interactions and resistance. Pharmacol Ther 1999;83(2):141-51.

[4] Endimiani A, Perez F, Bonomo RA. Cefepime: a reappraisal in an era of increasing antimicrobial resistance. Expert Rev Anti Infect Ther 2008;6(6):80524.

[5] Sharma S, Gupta A, Arora A. Cefepime Tazobactam: a new $\beta$ lactam/ $\beta$ lactamase inhibitor combination against ESBL producing gram negative bacilli. Int J Pharm Biomed Sci 2012;3(2):35-8.

[6] Nguyen HM, Shier KL, Graber CJ. Determining a clinical framework for use of cefepime and beta-lactam/betalactamase inhibitors in the treatment of infections caused by extended-spectrum-beta-lactamaseproducing Enterobacteriaceae. J Antimicrob Chemother 2014;69(4):871-80.

[7] Winn W. Konemann's Color atlas and diagnostic text of microbiology. $6^{\text {th }}$ edn. Philadelphia, PA: Lippincott Williams \& Wilkins Publishers 2006: p. 945-1021.

[8] Kirby WM, Yoshihara GM, Sundsted KS, et al. Clinical usefulness of a single disc method for antibiotic sensitivity testing. Antibiotics Annu 1956-1957;892-7.

[9] EUCAST 2016. Breakpoint tables for interpretation of MICs and zone diameters. Version 6.0, January 2016. http://www.eucast.org/clinical_breakpoints/.

[10] Bhat YR, Lewis LE, Vandana KE. Bacterial isolates of early-onset neonatal sepsis and their antibiotic susceptibility pattern between 1998 and 2004: an audit from a center in India. Ital J Pediatr 2011;37:32.
[11] Shrestha S, Shrestha NC, Singh DS, et al. Bacterial isolates and its antibiotic susceptibility pattern in NICU. Kathmandu Univ Med J 2013;11(41):66-70.

[12] Veeraraghavan B, Jesudason MR, Prakasah JAJ, et al. Antimicrobial susceptibility profiles of gram-negative bacteria causing infections collected across India during 2014-2016: study for monitoring antimicrobial resistance trend report. Indian J Med Microbiol 2018;36(1):32-6.

[13] Abdul KG, Vidyalakshmi PR, Jayalakshmi VA, et al. Susceptibility profile of Gram-negative bacteremic isolates to beta lactam-beta lactamase inhibitor agents in comparison to other antibiotics. Indian J Cancer 2014;51(4):450-2.

[14] Navaneethakrishnan RM, Sundaram M, Ismail M. Susceptibility of bacterial isolates to combination of third generation cephalosporins and sulbactams. J Evolution Med Dent Sci 2016;5(37):2173-7.

[15] Bhat V, Gupta S, Kelkar R, et al. Bacteriological profile and antibiotic susceptibility patterns of clinical isolates in a tertiary care cancer center. Indian J Med Paediatr Oncol 2016;37(1):20-4.

[16] Dalal P, Gathwala G, Gupta M, et al. Bacteriological profile and antimicrobial sensitivity pattern in neonatal sepsis: a study from North India. Int J Res Med Sci 2017;5(4):1541-5. 